\title{
COMPOSICION ALGAL Y BIOINDICADORES DE CALIDAD DE AGUA. CASO DE ESTUDIO: EMBALSE SAN ROQUE, CÓRDOBA. ARGENTINA
}

\section{ALGAL COMPOSITION AND WATER QUALITY BIOINDICATORS. CASE STUDY: SAN ROQUE RESERVOIR, CÓRDOBA. ARGENTINA}

\author{
Inés Claudia Daga ${ }^{(1)}$, María Cecilia Fernández Belmonte ${ }^{(2)}$, Santiago María Reyna ${ }^{(3)}$ \\ ${ }^{(1)}$ Facultad de Ciencias Exactas Físicas y Naturales, Universidad Nacional de Córdoba. Córdoba, Córdoba, Argentina. \\ ${ }^{(2)}$ Facultad de Ingeniería y Ciencias Agropecuarias, Universidad Nacional de San Luis. Villa Mercedes, San Luis, Argentina. \\ ${ }^{(3)}$ Facultad de Ciencias Exactas Físicas y Naturales, Universidad Nacional de Córdoba. Córdoba, Córdoba, Argentina.
}

${ }^{(*)}$ e-mail: inesclaudiadaga@gmail.com

\begin{abstract}
RESUMEN
El embalse San Roque se encuentra a $11 \mathrm{~km}$ de la ciudad de Carlos Paz y a 40 de la ciudad de Córdoba y una de las funciones es brindar agua a gran parte de la ciudad y sus alrededores. Debido a que recibe un alto aporte de nutrientes provenientes principalmente de la actividad antrópica constituye un ecosistema favorable para el desarrollo de comunidades algales. El objetivo general del trabajo fue evaluar el estado trófico del embalse a través de índices de eutrofización e indicadores biológicos. Se llevaron a cabo muestreos estacionales desde febrero de 2014 hasta febrero de 2016, en el muro de cierre de embalse y la desembocadura de los tributarios. Se midieron parámetros físicos y químicos y determinaron nutrientes. Se realizó la identificación taxonómica de las especies, se calculó la biomasa fitoplanctónica mediante el biovolumen y clorofila $a$ y se estimó el índice de estado trófico. Se determinaron un total de 204 Taxa. El componente dominante del fitoplancton fue el dinoflagelado Ceratium furcoides y como subdominante la diatomea Aulacoseira granulata. De acuerdo al índice de estado trófico ubicamos al embalse San Roque en estado eutrófico, condiciones indeseables en un ambiente utilizado para provisión de agua potable.
\end{abstract}

Palabras clave: Fitoplancton, embalses, eutrofización, bioindicadores, calidad de agua

\begin{abstract}
The San Roque reservoir is $11 \mathrm{~km}$ from the city of Carlos Paz and $40 \mathrm{~km}$ from the city of Córdoba and one of the functions is to provide water to much of the city and its surroundings. Because it receives a high contribution of nutrients mainly from anthropic activity, it constitutes a favorable ecosystem for the development of algal communities. The general objective of the work was to evaluate the trophic status of the reservoir through eutrophication indices and biological indicators. Seasonal samplings were carried out from February 2014 to February 2016, at the closure wall of the reservoir and the mouth of the tributaries. Physical and chemical parameters were measured and nutrients were determined. The taxonomic identification of the species was carried out, the phytoplankton biomass was calculated using the biovolume and chlorophyll a, and the trophic status index was estimated. A total of 204 Taxa were determined. The dominant component of phytoplankton was the dinoflagellate Ceratium furcoides and as a subdominant the Aulacoseira granulata diatom. According to the trophic status index, we located the San Roque reservoir in a eutrophic state, undesirable conditions in an environment used to provide drinking water.
\end{abstract}

Key words: Phytoplankton, reservoirs, eutrophication, bioindicators, water quality 


\section{INTRODUCCIÓN}

El embalse San Roque se encuentra ubicado en el centro oeste de la provincia de Córdoba en el valle de Punilla a $40 \mathrm{~km}$ aguas arriba de la ciudad capital de la provincia. Fue construido entre 1888 y 1944, principalmente para abastecimiento de agua, generación de energía eléctrica, riego y control de crecidas. En la actualidad es uno de los ambientes acuáticos continentales más problemáticos del país debido a su avanzado grado de eutrofización. El aporte de líquidos residuales provenientes de los asentamientos poblacionales e infraestructura turística en la proximidad del lago y en la cuenca, sin adecuado tratamiento, así como de las embarcaciones, contribuyen al deterioro de la calidad de las aguas del embalse, comprometiendo los usos del recurso. Además, el uso del suelo con destino agrícola, forestal, ganadero y el empleo de agroquímicos en el perilago y cuenca de aporte, se relacionan directamente con el aporte de nutrientes favoreciendo el crecimiento desmedido de algas, aumentando de este modo el nivel trófico y trayendo como consecuencia el deterioro de la calidad del agua (Reyna et al., 2005; Degano et al., 2016; Amé et al., 2017).

El primer trabajo en el área fue el de Guarrera (1948) es un estudio de la ficoflora y los parámetros físico químicos del embalse. A partir de la década del 70 comienzan las floraciones de cianobacterias que aumentaron en intensidad y frecuencia con los años (Cachi, 1975; García de Emiliani, 1977; Gavilán, 1981; Pizzolón et al., 1999; Amé et al., 2017). Durante el período 1999-2000 se observaron por primera vez dinoflagelado del género Ceratium (Ruibal Conti et al., 1999; Girbal et al., 2000) y desde su aparición se han detectado episodios de floraciones en numerosas ocasiones y las mismas están relacionadas con altos niveles de la cota y con un marcado grado de estratificación (Rodríguez et al., 2005, 2013).

\section{OBJETIVOS}

El objetivo del trabajo fue evaluar el estado trófico del embalse a través de las variables físicoquímicas y biológicas y analizar la composición y estructura de la comunidad algal en base a los análisis de abundancia, biomasa, clorofila, diversidad y riqueza específica.

\section{METODOLOGÍA}

Se realizaron 8 muestreos estacionales, durante el período comprendido entre febrero 2014 a febrero 2016. Se aclara que no fue posible tomar muestras durante el verano de 2015, debido al exceso de precipitaciones, cuando ambos embalses superaron la cota de vertedero, incluso hasta comienzos del otoño. Se consideraron cinco estaciones de monitoreo y fueron ubicadas en el paredón del embalse y en la desembocadura de los tributarios. Estación $\mathrm{E}_{1}$ : paredón de embalse San Roque; estación $\mathrm{E}_{2}$ : desembocadura río Cosquín; estación $\mathrm{E}_{3}$ : desembocadura arroyo Las Mojarras; estación $\mathrm{E}_{4}$ : desembocadura arroyo Los Chorrillos y estación $\mathrm{E}_{5}$ : desembocadura río San Antonio (Figura 1).

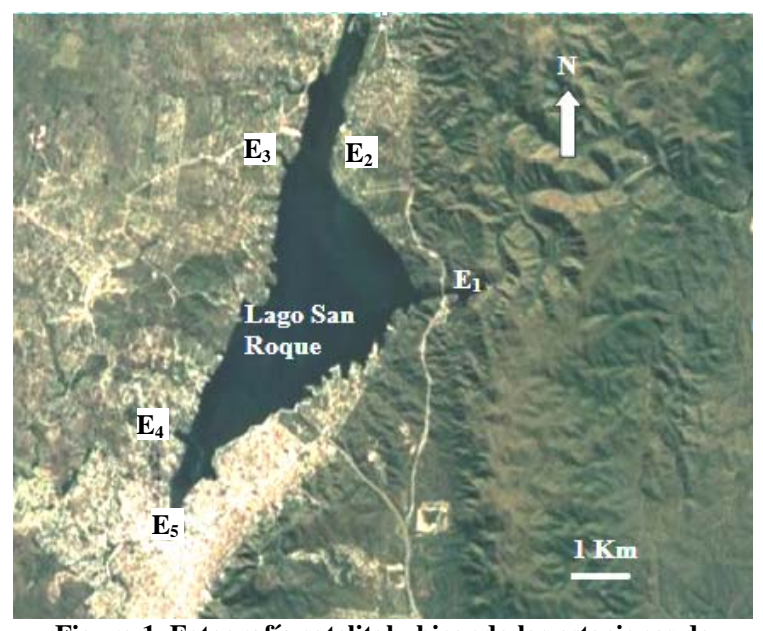

Figura 1. Fotografía satelital ubicando las estaciones de muestreo.

En el cierre del embalse $\left(\mathrm{E}_{1}\right)$ las muestras se tomaron con un muestreador tipo Van Dorn. Se realizó un perfil de tres profundidades en la columna de agua. La primera muestra se tomó a los 0.30 metros, la segunda a 1.5 metros de la superficie y la tercera a los 3 metros. En la desembocadura de los tributarios se utilizó una red de plancton de $20 \mu \mathrm{m}$ de diámetro de poro. Se obtuvieron muestras para identificación y análisis cualitativo y cuantitativo de fitoplancton, para clorofila y nutrientes. Para la medición de la transparencia se utilizó el Disco de Secchi y las variables fisicoquímicas (temperatura, $\mathrm{pH}$, conductividad eléctrica y oxígeno disuelto) se midieron in situ con instrumentos portátiles.

El análisis taxonómico se realizó mediante el empleo de microscopio óptico, las muestras fueron observadas in vivo y para la ubicación taxonómica 
de las especies se utilizaron las claves, manuales de identificación específica para cada grupo.

Para el recuento de organismos se utilizó la cámara de Sedgwick-Rafter. Se estimó la biomasa fitoplanctónica a partir de la clorofila a (APHA, 1992) y el biovolumen utilizando formas geométricas seleccionadas de modelos propuestos por Hillebrand et al., 1999; Sun \& Liu, 2003. Se estimó la frecuencia relativa a partir de la construcción de tablas de presencia-ausencia con todas las especies observadas durante los períodos muestreados se calcularon rangos de frecuencia de acuerdo al número de especies y porcentaje de frecuencia para cada lugar:

$$
F R=\frac{S_{i}}{N} \times 100
$$

donde $\mathrm{S}_{\mathrm{i}}$ es el número de presencia de la especie i en las muestras y $\mathrm{N}$ es el número total de muestras.

Para el cálculo de la diversidad se utilizó el índice de Shannon Weaver (1949). La evaluación del estado trófico se realizó mediante el Índice de Carlson (1977). Para el análisis estadístico de los datos se usó el programa InfoStat Software (Di Rienzo et al., 2017).

\section{ÁREA DE ESTUDIO}

El embalse San Roque se halla ubicado en las Sierras Chicas entre los meridianos $64^{\circ} 20^{\prime}$ y $64^{\circ} 27^{\prime}$ de longitud Oeste y los paralelos $31^{\circ} 14^{\prime}$ y $31^{\circ} 22^{\prime}$ de latitud Sur. Recibe el aporte de cuatro tributarios; ríos Cosquín, San Antonio y Los Chorrillos y el arroyo Las Mojarras y forma parte de la cuenca del río Suquía (Primero) que pertenece al gran sistema endorreico, que tiene como nivel de base la Laguna de Mar Chiquita. La precipitación media anual es de $780 \mathrm{~mm}$ y en la actualidad se observa una alternancia de años muy húmedos y otros secos. El embalse tiene un comportamiento que acompaña el régimen de lluvias, presentando niveles mínimos al final del invierno y valores máximos en época estival.

\section{RESULTADOS Y DISCUSIÓN}

Durante los últimos 100 años, la región central de Argentina se caracterizó por presentar escenarios hidrológicos contrastantes. La variabilidad hidroclimática asociada a la actividad del Sistema Monzónico Sudamericano y a los eventos de El Niño, entre otros, afecta profundamente la disponibilidad del recurso hídrico, tanto en cantidad como en calidad del agua, y controla la frecuencia e intensidad de fenómenos como sequías e inundaciones extremas (Pasquini et al., 2006; Piovano et al., 2009). Cuando se comenzó este estudio el nivel de la cota del embalse se encontraba 4 metros por debajo del nivel del vertedero, producto de una sequía prolongada en toda la provincia de Córdoba. Las abundantes precipitaciones registradas en el mes de febrero de 2014 llevaron al embalse a su cota máxima. Dicho nivel se conservó durante todo el periodo de estudio y las abundantes lluvias del verano de 2015 causaron inundaciones en las sierras chicas imposibilitando el muestreo durante ese verano.

De acuerdo al régimen térmico local, el embalse estudiado es de tipo monomíctico, presenta un solo periodo de mezcla que ocurre durante el invierno y se estratifica en verano (Rodríguez et al., 2013; Degano et al., 2016). En el presente trabajo los muestreos se realizaron hasta los $3 \mathrm{~m}$ de profundidad debido a ello solo se pudo observar una diferencia de temperatura de $1^{\circ} \mathrm{C}$ entre la muestra superficial y la muestra obtenida a los $3 \mathrm{~m}$. En la Tabla 1 se muestran los valores promedios de las determinaciones físicas y químicas de la $\mathrm{E}_{1}$ y en la Tabla 2 los valores promedios de las variables físicas y químicas en desembocadura de los tributarios.

Tabla 1. Valores promedios estacionales de los parámetros físicos químicos en la estación de muestreo paredón embalse

\begin{tabular}{|l|c|c|c|c|}
\hline \multicolumn{5}{|c|}{ San Roque $\left(\mathbf{E}_{\mathbf{1}}\right)$. } \\
\hline Vartaciones Año & $\mathrm{V}$ & $\mathrm{O}$ & $\mathrm{I}$ & $\mathrm{P}$ \\
\hline Disco de Secchi $(\mathrm{m})$ & 0.9 & 1.1 & 1.2 & 1.2 \\
\hline Temperatura $\left({ }^{\circ} \mathrm{C}\right)$ & 27 & 16 & 16 & 24 \\
\hline $\mathrm{pH}$ & 8.8 & 8.5 & 8.7 & 9.0 \\
\hline Conductividad $(\mu \mathrm{S} / \mathrm{cm})$ & 297 & 302 & 385 & 250 \\
\hline O.D. $(\mathrm{mg} / \mathrm{L})$ & 4.0 & 8.2 & 8.2 & 5.4 \\
\hline Nitrógeno $(\mathrm{mg} / \mathrm{L})$ & 0.89 & 1.43 & 1.43 & 1.07 \\
\hline Nitrato $(\mathrm{mg} / \mathrm{L})$ & 0.69 & 2.63 & 1.75 & 0.79 \\
\hline Nitrito $(\mathrm{mg} / \mathrm{L})$ & 0.04 & 0.01 & 0.02 & 0.02 \\
\hline Fósforo $(\mathrm{mg} / \mathrm{L})$ & 0.28 & 0.20 & 0.98 & 1.96 \\
\hline
\end{tabular}

En este trabajo las concentraciones de nitratos disminuyeron con las precipitaciones posiblemente 
por dilución. Refuerza esta posible explicación las correlaciones negativas entre nitratos y precipitaciones el análisis de la varianza fue significativo $(\mathrm{r}=-0.79 ; \mathrm{p}=0.03)$.

Los valores de nitritos fueron bajos lo que puede estar relacionado con los niveles de oxígeno disuelto que generalmente fueron elevados, lo cual pudo favorecer la oxidación de los nitritos, que de esta manera pasan a nitratos por la presencia de bacterias nitrificantes, muy comunes en estos cuerpos de agua. En aguas superficiales, bien oxigenadas, los niveles de nitrito no suelen superar $1 \mathrm{mg} / \mathrm{L}$ (Stumm \& Morgan, 1981).

Tabla 2. Valores promedios estacionales de parámetros físicos y químicos, en la desembocadura de los tributarios $\left(E_{2}, E_{3}, E_{4}\right.$ y $\left.E_{5}\right)$.

\begin{tabular}{|c|c|c|c|c|c|c|c|c|c|c|c|c|c|c|c|c|}
\hline $\begin{array}{c}\text { Estaciones de } \\
\text { muestreo }\end{array}$ & \multicolumn{4}{|c|}{$\mathrm{E}_{2}$} & \multicolumn{4}{|c|}{$\mathrm{E}_{3}$} & \multicolumn{4}{|c|}{$\mathrm{E}_{4}$} & \multicolumn{4}{|c|}{$\mathrm{E}_{5}$} \\
\hline Eariables & V & $\mathrm{O}$ & I & $\mathrm{P}$ & V & $\mathrm{O}$ & I & $P$ & V & $\mathrm{O}$ & I & $P$ & V & $\mathrm{O}$ & I & $\mathrm{P}$ \\
\hline Temperatura $\left({ }^{\circ} \mathrm{C}\right)$ & 28 & 17 & 19 & 27 & 29 & 17 & 19 & 27 & 29 & 17 & 22 & 27 & 28 & 16 & 22 & 28 \\
\hline $\mathrm{pH}$ & 8.9 & 8.5 & 8.5 & 8.6 & 8.4 & 8.5 & 8.8 & 8.7 & 9.0 & 8.8 & 9.0 & 9.4 & 8.7 & 8.5 & 8.7 & 8.8 \\
\hline O.D. (mg/L) & 3.9 & 8.2 & 7.8 & 5.3 & 3.8 & 8.3 & 8.4 & 5.3 & 3.8 & 8.0 & 8.2 & 5.2 & 4.4 & 8.2 & 7.8 & 5.4 \\
\hline
\end{tabular}

La transparencia del agua (Tabla 1) fue menor durante los muestreos de verano y se observó una correlación positiva entre el número de células y biovolumen de diatomeas y el disco de Secchi $(r=0.80 ; p=0.017)$ lo que nos podría indicar que la transparencia del agua no se ve influenciada por la presencia de las diatomeas. En un estudio en el embalse Paso de Las Piedras encontró una correlación positiva entre transparencia y biovolumen donde las especies dominantes eran diatomeas (Fernández, 2010). Con C. furcoides si bien se observó una correlación negativa entre la abundancia y el disco de Secchi esta no fue significativa $(r=-0.28 ; p=0.50)$. Por lo que se infiere que el tamaño de las células que conforman el fitoplancton podría influir en la transparencia.

El pH fue elevado (Tablas 1 y 2) el embalse y sus tributarios se caracterizaron por presentar aguas alcalinas con un rango de variación de $\mathrm{pH}$ menor que en otros trabajos realizados en la misma área de estudio (García de Emiliani, 1977; Rodríguez et al., 2000, Daga y Pierotto, 2011). Las mayores tasas de fotosíntesis fitoplanctónica podrían ser responsables de los altos valores de $\mathrm{pH}$ observados (Rodríguez et al., 2013).

La conductividad presentó valores mayores en la desembocadura del rio Cosquín, $\mathrm{E}_{2}$ (Tabla 2). Este tributario presenta altos contenidos de carbonatos que provienen principalmente de la disolución de minerales carbonáticos (calcita, dolomita, etc.) y silicatos presentes en rocas y suelos de la subcuenca (Gaiero, 1998). En este tributario se observó la presencia de especies que no prosperan en aguas con bajo contenido de carbonato de calcio como la diatomea Gomphonema olivaceum var. calcarea y el alga verde Phacotus lenticularis (Winsborough y Golubic, 1987, Schlegel et al., 1998, Gruenert y Raeder, 2014).

Las concentraciones de oxígeno disuelto en todas las estaciones de muestreo presentaron un patrón de variación temporal que se explica en relación a la solubilidad de este gas con la temperatura, aumentando considerablemente al disminuir la misma (Tablas 1 y 2). Se observa que los valores son mayores en otoño-invierno y más bajos en primavera-verano. Idéntica situación fue registrada en otros trabajos realizados en la misma área (García de Emiliani, 1977, Daga y Pierotto 2011).

En el embalse y sus tributarios se registraron 204 taxones distribuidos en nueve grupos, las Diatomea presentaron el mayor número de especies (85), en segundo lugar las Chloroplastida (66), continuan luego las Cyanobacteria (27), Euglenozoa (17), Chrysophyceae (4), Cryptophyceae (2), Rhodophyceae (1), Xanthophyceae (1) y Dinophyceae (1).

La estación de muestreo con mayor número de especies fue $\mathrm{E}_{5}$, con un promedio de 31 especies y el menor número de especies ocurrió en $\mathrm{E}_{1} \mathrm{y}_{4}$, con un promedio de 8 especies. Un análisis de la varianza no paramétrica (prueba Kruskal Wallis) para la riqueza de especies, permitió agrupar las estaciones de muestreo $E_{1} \mathrm{y}_{4}$ por un lado y $E_{2}, E_{3} y$ 
$\mathrm{E}_{5}$ por el otro presentando diferencias significativas entre los dos grupos $(\mathrm{H}=26.91 ; \mathrm{p}=0.0001)$.

Dentro de los resultados obtenidos las diatomeas presentaron la mayor riqueza de especies. Los taxones más frecuentes para $\mathrm{E}_{1}$ fueron las diatomeas centrales como Aulacoseira granulata con una frecuencia del $100 \%$. Esta especie está citada como abundante para numerosos embalses como el de Salto Grande (De León y Chalar, 2003), el embalse de Río Hondo (Subsecretaria de Recursos Hídricos, 2008) y Yacyretá (Meichtry de Zamburlín et al., 2013) y para tributarios del rio Paraná (O'Farrell \& Tell, 2001; García de Emiliani \& Devercelli, 2004).

Otra diatomea representativa para $\mathrm{E}_{1}$ fue Cyclotella meneghiniana presentó una frecuencia del $40 \%$. Especie que tolera un alto rango de conductividad y es común en ambientes acuáticos con abundante materia orgánica (Isra de Alcántara et al., 2002, Ortega Murillo et al., 2010).

En la desembocadura de los tributarios las especies que presentaron mayor frecuencia fueron Ulnaria ulna con el $80 \%$, Navicula gregaria con el $60 \%$ y Melosira varians con una frecuencia del $50 \%$. Las tres especies mencionadas junto a Gomphonema parvulum, Fragilaria construens, Navicula cryptocephala y Nitzschia palea son consideradas tolerantes a la polución y buenas indicadoras de eutrofización (Sabater et al., 1988; Morales, 2016).

Las Chloroplastida presentaron una mayor riqueza de especies en las desembocaduras de los tributarios con un máximo desarrollo en primavera y verano, donde las condiciones ambientales como la poca profundidad, largos períodos de luz solar y temperaturas relativamente altas favorecieron su desarrollo (Luque y Martínez de Fabricius, 2003). Se identificaron numerosas especies unicelulares y coloniales citadas para cuerpos de agua con altos niveles de eutrofia como Monoraphidium griffithii; Pediastrum boryanum (Temponeras et al., 2000); Actinastrum hantzschii, Monactinus simplex y Coelastrum reticulatum (González et al., 2003; Becerra, 2009) y Nephrocytium agardhianum, Staurastrum leptocladum y Closterium aciculare (Canosa y Pinilla, 1998; Pinilla, 2000).

Las algas verdes filamentosas representadas por Cladophora glomerata, Chaetomorpha herbipolensis, Ulothrix sp., Chaetophora sp. y Oedogonium sp., generalmente se desarrollan adheridas a algún tipo de sustrato y su presencia en el plancton se debe al efecto de la deriva procedente del bento como consecuencia de la acción de la velocidad de corriente (Biasotti et al., 2014; Galea et al., 2014).

Las Cyanobacteria ocuparon el tercer lugar en número de especies, la mayoría de los taxones son filamentosos, De León y Chalar (2003) afirman que la dominancia de formas filamentosas sobre las coloniales, indican condiciones turbulentas del sistema, situación acorde al período de estudio.

Se identificaron 24 géneros de cianobacterias de los cuales 13 son reportados en la literatura como productores de cianotoxinas: Dolichospermun, Gomphosphaeria, Limnothrix, Lyngbya, Merismopedia, Microcystis, Oscillatoria, Phormidium, Planktothrix, Pseudoanabaena, Synechocystis, Synechococcus y Woronichinia (De León, 2002; Bonilla, 2009; Gianuzzi, 2011; Aguilera et al., 2018).

Dentro de Euglenozoa Trachelomonas volvocina presentó la mayor frecuencia $(15 \%)$, se la puede encontrar en todo tipo de ambiente, sin embargo es más representativa en aguas mesotróficas a eutróficas, por lo que se considera bioindicadora de altos niveles de trofía (Pinilla, 2000; RodríguezZambrano y Aranguren-Riaño, 2014).

Se identificó una especie de Rhodophyceae, Compsopogon coeruleus su presencia en la parte baja de los tributarios es por arrastre después de las crecidas (Carmona, 2012). En Córdoba dicha especie ha sido citada en dos hábitats bien diferentes, en arroyos serranos de aguas transparentes y baja conductividad y para un lago urbano con tendencia a eutrofizarse en el verano y altos valores de conductividad (Leyes et al., 2018).

Las Chrysophyceae fueron de aparición esporádica a excepción de Anthophysa vegetans que estuvo presente en todas las estaciones del año a excepción del verano. Las Cryptophyceae, flageladas adaptadas a las bajas temperaturas y a la escasez de luz se identificaron solo en $\mathrm{E}_{1}$.

El grupo de Dinophyceae estuvo representado por un solo taxón: Ceratium furcoides. Para ambientes de agua dulce se reportan 6 especies de Ceratium donde las habituales son $C$. hirundinella y $C$. furcoides (Popovsky y Pfiester, 1990). Ambas especies a partir de la década del 90 comenzaron a expandirse por Sudamérica (Guerrero y Echenique, 1997; Boltovskoy et al., 2013; Silva et al., 2012). 
En los últimos años $C$. furcoides desplazó a $C$. hirundinella, probablemente un aumento de nutrientes y el cambio climático fueron los principales factores que contribuyen al establecimiento de $C$. furcoides (Meichtry de Zaburlin et al., 2014; Cavalcante et al., 2016). Almanza et al., (2016) sugieren que la plasticidad fenotípica de $C$. furcoides podría favorecer la capacidad invasora de este organismo, proporcionando una ventaja adicional para generar floraciones, así como para establecerse y dominar en el nuevo hábitat. Ceratium no produce toxinas pero genera numerosos impactos en el recurso hídrico, como modificar las propiedades organolépticas del agua, dificultar $\mathrm{y} / \mathrm{o}$ encarecer los procesos de potabilización debido a la gran movilidad y tamaño, los organismos decantados permanecen activos, nadan arrastrando parte del floc (decantador), lo que produce un aumento en la turbiedad del agua disminuyendo la eficacia del decantador. Pueden llegar hasta la superficie siendo arrastrado hacia los filtros provocando el taponamiento prematuro de los mismos (Girbal et al., 2000).

El número de células de fitoplancton no presentó diferencias significativas al analizar las muestras tomadas en distinta profundidad en la estación $E_{1}$
(Prueba de Kruskal-Wallis, $\mathrm{H}=4.64 ; \mathrm{p}=0.098$ ). Si se observaron diferencias significativas para el número de células entre $E_{1}$ y las desembocaduras de los tributarios, $E_{2}, E_{3}, E_{4}$ y $E_{5}(H=15.54 ; p=0.0037)$ como también el biovolumen presentó diferencias significativas entre $\mathrm{E}_{1} \mathrm{y}$ el resto de los tributarios $(\mathrm{H}=22.1 ; \mathrm{p}=0.0002)$. La mayor abundancia celular en $\mathrm{E}_{1}$ ocurrió durante el invierno de 2014 y fue de 10850 cél $/ \mathrm{mL}$ con un biovolumen de $55 \mathrm{~mm}^{3} / \mathrm{L}$ (Figura 2). La diatomea Aulacoserira granulata fue dominante con 7000 cél $/ \mathrm{mL}$, las células restantes corresponden a otras diatomeas y a $C$. furcoides.

En los muestreos restantes se observaron entre 500 a 5600 cél $/ \mathrm{mL}$, con biovolumenes entre 12 y 146 $\mathrm{mm}^{3} / \mathrm{L}$. Los mayores valores de biovolumen se corresponden con mayores abundancias de Ceratium. Al estudiar la dinámica de las poblaciones fitoplanctónicas se considera que es de gran importancia tener en cuenta el número de células y el biovolumen, ya que un análisis basado sólo en el número de células podría exagerar la importancia de las formas pequeñas, mientras que un análisis basado sólo en el biovolumen puede aumentar la importancia de las formas de gran tamaño (Fernández, 2010).

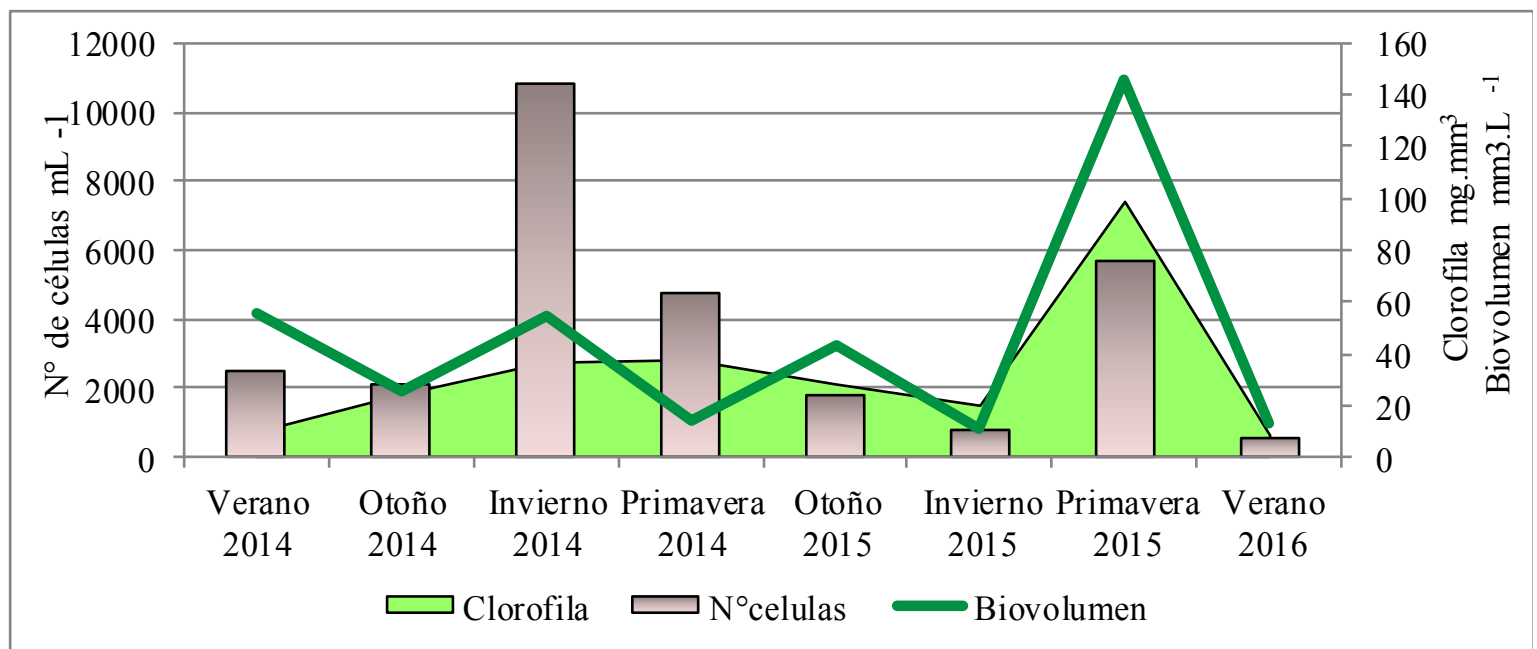

Figura 2. Variación del número de células, biovolumen y clorofila a en la estación paredón embalse San Roque ( $\left.E_{1}, 0.30 \mathrm{~m}\right)$ durante el período muestreado.

En la desembocadura de los tributarios el número de células estuvo comprendido entre 50 a 1700 cél $/ \mathrm{mL}$ y biovolumen entre 0.07 y $12 \mathrm{~mm}^{3} / \mathrm{L}$. A excepción del muestreo del verano de 2016 en la desembocadura del arroyo Los Chorrillos $\left(\mathrm{E}_{4}\right)$, donde se registraron 15000 cél $/ \mathrm{mL}$, de $C$. furcoides. Esta distribución irregular puede deberse a la acción del viento que llevó todos los organismos hacia $\mathrm{E}_{4}$, donde el agua adquirió un color marrón rojizo y un fuerte olor a pescado. Situación semejante fue observada en el embalse Escaba de Tucumán donde el mayor número de Ceratium hirundinella se registró en el río Singuil, tributario del embalse Escaba y atribuyen este aumento a una influencia del viento (Martinez de Marco et al., 2018). 
El biovolumen de los tributarios (a excepción del valor extraordinario del verano de 2016 para $\mathrm{E}_{4}$ ) se encuentra entre los valores mencionados para una revisión de unos 50 ríos del mundo (81\% templados y $19 \%$ tropicales) con valores promedios entre 0.06 y $25 \mathrm{~mm}^{3}$ (Rojo et al., 1994, tomado de Zalocar, 1999).

Se observaron cianobacterias productoras de toxinas de los géneros Planktothrix y Limnotrix en las desembocaduras de los tributarios $\mathrm{E}_{4}$ y $\mathrm{E}_{5}$ con biovolúmenes de $0.006 \mathrm{~mm}^{3} / \mathrm{L}$ y de $0.03 \mathrm{~mm}^{3} / \mathrm{L}$. La Organización Mundial de la Salud establece Niveles Guía de biovolúmenes para cianobacterias potencialmente productoras de toxinas (Chorus y Bartram, 1999) donde valores entre 0.02 a 0.2 $\mathrm{mm}^{3} / \mathrm{L}$ indicarían un nivel de vigilancia aconsejando aumentar la frecuencia de muestreos.

La clorofila $a$ en la estación $\mathrm{E}_{1}$ presentó el mínimo valor en el verano de 2016 coincidiendo con el menor número de células y el menor valor de biovolumen. El máximo valor de clorofila se registró en la primavera de 2015 donde también se registró el máximo valor de biovolumen.

Los mayores valores de clorofila $a$ se corresponden con altas densidades de Ceratium, si bien no hay una relación lineal entre el tamaño celular y el contenido de clorofila $a$, los organismos de mayor volumen celular tienen un contenido de clorofila $a$ mayor que los organismos pequeños y contribuyen en mayor proporción a la productividad neta del ecosistema (Felip y Catalán, 2000). La clorofila $a$ presentó una correlación positiva con el número de células $(\mathrm{r}=$ $0.76 ; \mathrm{p}=0.043)$.

Las dimensiones de $C$. furcoides no fue la misma a lo largo de todo el estudio. Al realizar las mediciones de varios organismos se pudo observar que cuando la abundancia de $C$. furcoides fue mayor las células tenían menor tamaño (Media del largo celular $=185 \mu \mathrm{m}$; Media del ancho $=30 \mu \mathrm{m})$. Por el contrario, cuando la abundancia fue menor, el tamaño de C. furcoides se incrementó (Media del largo celular $=190 \mu \mathrm{m}$; Media del ancho $=41 \mu \mathrm{m}$ ). La diversidad se calculó a través del índice de Shannon \& Weaver y presentó valores bajos $(<\mathrm{a} 1)$ en el embalse propiamente dicho $\left(\mathrm{E}_{1}\right)$ debido a la desproporcionada representación numérica de una especie dominante y subdominancia de otras pocas especies, así como una reducida abundancia para la mayoría de los taxones. Resultados semejantes fueron observados para el San Roque hace poco más de una década (Prósperi et al., 2007), mientras que en un estudio realizado hace más de cuarenta años para el mismo embalse la diversidad oscilaba entre 1.5 y 3 bits (García de Emiliani, 1977). Una reducción de la diversidad es un excelente indicador de contaminación (Patrick et al., 1949) ya que la polución rompe un equilibrio preexistente $\mathrm{y}$ la comunidad se simplifica. Disminuye la riqueza de especies, pero algunas de ellas pueden estar representadas por un número elevado de individuos. En tal sentido, Sournia (1978) señaló que la diversidad disminuye cuando los individuos de especies raras son sustituidos por individuos de especies que ya eran comunes.

El análisis del estado trófico se efectuó a través del TSI (Trophic Status Index), el cual tiene en cuenta la concentración de clorofila $a$, la concentración de fósforo total y la transparencia del agua medida con el disco de Secchi. Los valores del TSI para el disco de Secchi oscilaron entre 59 a 68, para el fósforo, estuvieron comprendidos entre 69 a 115 y para la clorofila entre 48 a 75 (Tabla 3 ).

Tabla 3. Variación del estado trófico del embalse San Roque.

\begin{tabular}{|c|c|c|c|c|c|}
\hline $\begin{array}{l}\text { ESTADO } \\
\text { TROFICO } \\
\end{array}$ & TSI & \begin{tabular}{|c|} 
ESTACIONES \\
DELAÑO \\
\end{tabular} & $\begin{array}{l}\text { TSIDISCO } \\
\text { SECCHI (m) } \\
\end{array}$ & $\begin{array}{l}\text { TSI P } \\
\left(\mathrm{mg} \mathrm{m}^{-3}\right) \\
\end{array}$ & $\begin{array}{l}\text { TSIClorofila } \\
\left(\mathrm{mg} \mathrm{m}^{3} 3\right.\end{array}$ \\
\hline Oligotrófico & $0-30$ & & & & \\
\hline Mesotrófico & $30-60$ & & & & \\
\hline \multirow[t]{8}{*}{ Eutrófico } & $60-90$ & Verano 2014 & 68 & 69 & 49 \\
\hline & & Otoño 2014 & 64 & 69 & 60 \\
\hline & & \begin{tabular}{|l|} 
Invierno 2014 \\
\end{tabular} & 59 & 100 & 66 \\
\hline & & $\begin{array}{l}\text { Primavera } \\
2014 \\
\end{array}$ & 61 & 69 & 65 \\
\hline & & Otoño 2015 & 64 & 83 & 61 \\
\hline & & Invierno 2015 & 59 & 94 & 57 \\
\hline & & $\begin{array}{c}\text { Primavera } \\
2015 \\
\end{array}$ & 67 & 115 & 75 \\
\hline & & Verano 2016 & 68 & 88 & 48 \\
\hline Hipereutrófico & $>90$ & & & & \\
\hline
\end{tabular}

De acuerdo a los valores del disco de Secchi en el $75 \%$ de los muestreos clasificamos al embalse como eutrófico y el $25 \%$ restante como mesotrófico. De acuerdo al fósforo en el $62 \%$ de los muestreos el embalse se encuentra eutrófico con tendencia a hipertrófico. Teniendo en cuenta la clorofila en el $50 \%$ de los muestreos el embalse se encuentra en estado mesotrófico y el $50 \%$ restante eutrófico.

Altos valores del índice de estado trófico calculados a partir de la concentración de fósforo total en relación a las otras variables también apoyarían el hecho que el fitoplancton probablemente esté 
limitado por otros factores más que por la concentración de fósforo (Fernández, 2010). Carlson (1977) sugiere que, para los fines de clasificación del estado trófico, se le dé prioridad a parámetros biológicos tales como la clorofila.

En coincidencia con Fernández-Cirelli (1999) la eutrofización debe analizarse desde el punto de vista de sus causas y de sus consecuencias, ya que termina siendo un problema para la salud de la población.

\section{CONCLUSIONES}

Se determinaron un total de 204 Taxa donde el componente dominante fue el dinoflagelado Ceratium furcoides y como subdominante la diatomea Aulacoseira granulata.

La elevada biomasa fitoplanctonica la baja diversidad, los cambios de coloración y el estado de eutrofia son características que indican un significativo deterioro de la calidad del recurso hídrico.

Dado que el uso principal es la provisión de agua para consumo se considera que es importante planificar actividades concretas de control de eutrofización y para ello se hará necesario diseñar un plan de manejo adecuado.

\section{REFERENCIAS}

Aguilera, A., Haakonssonb, S., Martina, M. V., Salerno, G. L. y Echenique, R. (2018). Bloomforming cyanobacteria and cyanotoxins in Argentina: A growing health and environmental concern. Limnologica, 69:103-114.

Almanza, V., Bicudo, C. M., Parra, O. y Urrutia, R. (2016). Características morfológicas y limnológicas de las floraciones de Ceratium furcoides (Dinophyta) en un lago somero de Chile Central. Limnetica, 35 (1): 253-268.

Amé, V., Ferral, A. y Solís, V. (2017). Eutrofización en el embalse San Roque y floraciones masivas de cianobacterias. Seguimiento por técnicas geoespaciales. UNCiencia. http://unciencia.unc.edu.ar/2017.

APHA. (1992). Standard Methods for the Examination of Waters and Wastewaters, 19th edn. APHA/AWWA/WPCF, Washington, DC. P. 1193.
Becerra, C. L. E. (2009). Catálogo fitoplanctónico de los lagos del Dique, municipio de Xalapa, Veracruz, México (Tesis de licenciatura). Universidad Veracruzana: Veracruz, México.

Biasotti, A. E, Álvarez, S. B., Bazán, G. I. y Martínez de Fabricius, A. L. (2014). Variación estacional de la comunidad microfitoplanctónica del curso medio del Río Colorado (La PampaArgentina). Biología Acuática, 30: 249-258.

Boltovskoy, A., Echenique, R. y Guerrero, J. M. (2013). Colonización de limnotopos de Argentina por Ceratium hirundinella (Dinophyceae). Memorias XXIX Jornadas Argentinas de Botánica y XV Reunión Anual de la Sociedad Botánica de Chile. Boletín de la Sociedad Argentina de Botánica, 38 (Supl.).

Bonilla, S. (2009). Cianobacterias planctónicas del Uruguay. Manual para la identificación y medidas de gestión (No. 582.232 UNE). UNESCO. Administración de las Obras Sanitarias del Estado (Uruguay). Montevideo. Universidad de la República.

Cachi, J. (1975). Variaciones Planctónicas del Embalse San Roque y su relación con los procesos de potabilización. O.S.N., 26 pp.

Canosa, A. y Pinilla, G. (1998). Evaluación de la población bacteriana total en tres cuerpos de agua lénticos mediante la técnica de epifluorecencia. Informe final de proyecto. Colciencias, Universidad Jorge Tadeo Lozano. Bogotá. 60 pp.

Carlson, R. E. (1977). A trophic state index for lakes. Limnology Oceanography, 22: 361-369.

Carmona Jiménez, J. (2012). Las algas rojas (Rhodophyta) de agua dulce en la región central de México. Taxonomía y distribución. Monografías ficológicas V. 4.

Cavalcante, K. P., Cardoso, L. S., Sussella, R. y Becker, V. (2016). Towards a comprehension of Ceratium (Dinophyceae) invasion in Brazilian freshwaters: autecology of C. furcoides in subtropical reservoirs. Hydrobiologia, 771(1):265280.

Chorus, I. y Bartram, J. (1999). Toxic cyanobacteria in water: a guide to their public health consequences, monitoring and management / edited by Ingrid Chorus and JamieBertram. WorldHealth

Organization.https://apps.who.int/iris/handle/1066 $5 / 42827$

Daga, C. y Pierotto, M. (2011). Cianobacterias de la cuenca del Embalse San Roque. Boletín Sociedad Argentina de Botánica, 46 (3-4): 227-234.

Degano M., Rodríguez, M. I. y Dasso C. (2016). Dinámica térmica de cuerpos de agua eutróficos: 
caso Embalse San Roque. Revista Facultad de Ciencias Exactas, Físicas y Naturales. V 3(1): 93100.

De León, L. (2002). Floraciones de cianobacterias en aguas continentales del Uruguay: causas y consecuencias. En: Domínguez, A. \& Prieto, R. G. (eds.). Perfil ambiental del Uruguay 2002. Nordan Comunidad, Montevideo: 28-37.

De León, L. y Chalar, G. (2003). Abundancia y diversidad del fitoplancton en el embalse de Salto Grande (Argentina - Uruguay). Ciclo estacional y distribución espacial. Limnetica, 22 (1-2): 103113.

Di Rienzo, J. A., Casanoves, F., Balzarini, M. G., González, L., Tablada, M. y Robledo, C. W. (2017). Grupo InfoStat, FCA, Universidad Nacional de Córdoba, Argentina. URL http://www. infostat.com.ar.

Felip, M. y Catalan, J. (2000). The Relationship Between Phytoplankton Biovolume and Chlorophyll in a Deep Oligotrophic Lake: Decoupling in Their Spatial and Temporal Maxima. Journal of Plankton Research, 22(1):91105.

Fernández Cirelli, A. (1999). Aprovechamiento y Gestión de Recursos Hídricos. Programa Iberoamericano de Ciencia y Tecnología para el Desarrollo.

Fernández, C. (2010). Caracterización limnológica de un ambiente eutrófico: el embalse Paso de las Piedras, Argentina. Bioecología del fitoplancton. (Tesis de Doctorado en Biología). Universidad Nacional del Sur. Bahía Blanca.

Gaiero, D. (1998). Hidrogeoquímica de un sistema de la región semiárida: el Río Suquía, Córdoba, 2. Transporte de sólidos disueltos, meteorización y consumo de CO2. Revista de la Asociación Geológica Argentina, 53(3): 337:347.

Galea, M. J., Bazán, G. I., Álvarez, S. B. y Martínez De Fabricius, A. L. (2014). Estudio del fitoplancton aguas arriba y aguas abajo del Dique Embalse Casa de Piedra, río Colorado (La Pampa, Argentina). Biología Acuática, 30: 287-300.

Garcia de Emiliani, M. O. (1977). Ciclo Anual del Fitoplancton en el Embalse San Roque (Córdoba, Argentina). Revista de la Asociación de Ciencias Naturales del Litoral, 8:1-12.

Garcia de Emiliani, M. O. y Devercelli, M. (2004). Estructura y dinámica del fitoplancton de un rio tributario (Salado) y cauces secundarios del rio Paraná (Santa Fé, Coronda y El Vado) en el área de confluencia (Santa Fé, Argentina). Revista FABICIB, 8: 23-42.

Gavilán, J. G. (1981). Study of Water Quality in the
San Roque Reservoir. Water Quality Boulletin, Environment Canadá, 6(4): 136-158.

Gianuzzi, L. (2011). Cianobacterias como Determinantes Ambientales en la Salud, 1a ed., Buenos Aires, Ministerio de Salud de la Nación.

Girbal, A., Lammel, E. y Busso, F. (2000). Utilización de permanganato de potasio para mitigar un bloom de Ceratium hirundinella. Resúmenes del Seminario Internacional: Identificación y Control de Algas en la Producción de Agua Potable.

González, E. J., Ortaz, M., Peñaherrera, C., Montes, E., Matos, M. L. y Mendoza, J. (2003). Fitoplancton de cinco embalses de Venezuela con diferentes estados tróficos. Limnetica, 22 (1-2): 15-35.

Gruenert, U. y Raeder, U. (2014). Growth responses of the calcite-loricated freshwater phytoflagellate Phacotus lenticularis (Chlorophyta) to the $\mathrm{CaCO} 3$ saturation state and meteorological changes. Journal of Plankton Research, 36(3): 630-640.

Guarrera, S. (1948). El fitoplancton del embalse San Roque. Revista del Museo. Argentino de Ciencias. Naturales "Bernardino Rivadavia", 1 (2): 29-55.

Guerrero, J. M. y Echenique, R. O. (1997). Ceratium hirundinella blooms in Argentine reservoirs. Harmful Algae News 16: 3.

Hillebrand, H., Durselen, C. D., Kirschtel, U., Pollingher, T. y Zohary, T. (1999). Biovolume calculation for pelagic and benthic microalgae. Journal of Phycology, 35: 403-424.

Isra de Alcántara, I., Garduño-Monroy, V. H. y Ortega-Murillo, R. (2002). Paleoambiente lacustre del Cuaternario tardío en el centro del lago de Cuitzeo. Hidrobiologica, 2: 61-78.

Leyes, C., Bustamante, A., Giovanola, C. y Daga, C. (2018). Primer registro de Compsopogon caeruleus (Rhodophyta, Compsopogonales) en Córdoba (Argentina). Lilloa, 55(2): 67-74.

Luque, M. E. y Martínez de Fabricius, A. L. (2003). Distribución temporal del fitoplancton y epiliton en el río Piedra Blanca (Córdoba, Argentina). Limnetica, 22(3-4): 19-34.

Martínez de Marco, S. N., Tracanna, B. C., Isasmendi, S. C., Alderete, M del C., Taboada, M. de los A. y Mirande, V. (2018). Evaluación del fitoplancton en el embalse Escaba (Tucumán Argentina) para caracterizar la calidad de sus aguas. Boletín de la Sociedad Argentina de Botánica, 53 (4): 521-542.

Meichtry de Zaburlin, N., Vogler, R. E., Llano, V. M. y Martens, I. S. M. (2013). Fitoplancton del embalse Yacyretá (Argentina Paraguay) a una década de su llenado. Revista Mexicana de 
Biodiversidad, 84(1): 225-239

Meichtry de Zaburlin, N., Boltovskoy, A., Costigliolo Rojas, C. y Rodríguez, R. (2014). Primer registro del dinoflagelado invasor Ceratium furcoides (Levander) -Langhans 1925 en la Argentina y su distribución en el área de influencia del Embalse Yacyretá (río Paraná, ArgentinaParaguay). Limnetica, 33 (1): 153-160.

Morales, E. A. (2016). Floración de Ceratium furcoides (Levander) Langhans (Dinoflagellata, Dinophyceae) en la represa de La Angostura, Cochabamba, Bolivia. Acta Nova, 7(4):389-398.

O'Farrell, I. y Tell, G. (2001). Morphological variability of Aulacoseira granulata (Ehr.) Simonsen (Bacillariophyceae) in the Lower Paraná River (Argentina). Limnology, 2(2):65-71.

Ortega-Murillo, M. R., Alvarado-Villanueva, R., Hernández Morales, R. y Sánchez-Heredia, J. D. (2010). Evolución trófica de un lago tropical hiposalino en México con base al fitoplancton. Revista Biológicas, 12: 75-81.

Pasquini A. I., Lecomte, K. L., Piovano E. y Depetris P. J. (2006). Recent rainfall and runoff variability in central Argentina. Quaternary International, 158: 127-139.

Patrick, R. (1949). A proposed biological measure of stream conditions, based on a survey of the Conestoga Basin, Lancaster Country, Pennsylvania. Proc. Acad. Nat. Sc. Philadelphia. 101: 227- 341.

Pinilla, G. A. (2000). Indicadores biológicos en ecosistemas acuáticos continentales de Colombia. Bogotá. Fundación Universidad de Bogotá Jorge Tadeo Lozano.

Piovano, E., Ariztegui, D., Córdoba, F., Cioccale, M. y Sylvestre, F. (2009). Hydrological variability in South America below the Tropic of Capricorn (Pampas and eastern Patagonia, Argentina) during the last 13.0 ka. En: Vimeux. F., Sylvestre, F., Khodri, M. (Eds.) Past climate variability from the Last Glacial Maximum to the Holocene in South America and Surrounding regions: From the Last Glacial Maximum to the Holocene). Springer Developments in Paleoenvironmental Research Series (DPER), 323-351.

Pizzolón, L., Tracanna, B., Prósperi, C. y Guerrero, J. (1999). Cianobacterial blooms in argentinean inland water. Lakes and Reservoirs: Research \& Management, 4: 101-105.

Popovsky, J. y Pfiester, L. A. (1990). Dinophyceae (Dinoflagellida). In: Ettl, H., Gerloff, J., Heynig, H. and Mollenhauer, D., Süsswassserflora von Mitteleropa. Begründet von A. Pascher. Band 6 . Jena, Gustav Fischer Verlag.
Prósperi, C., Pierotto, M., Daga, C. y Rincón, A. (2007). Monitoreo de cianobacterias toxicas en embalses de Córdoba (Programa Conapris). Memorias V Taller Internacional sobre enfoques regionales para el desarrollo y gestión de embalses en la cuenca del Plata. Itaipú .Brasil- Paraguay.

Reyna, S., Orso, M., Reyna, E., Lábaque, M. y Gómez, C. (2005). Plan de gestión de los recursos hídricos de la provincia de Córdoba. Subsecretaria de Recursos Hídricos.

Rodríguez, M. I., Cossavella, A., Oroná, C., Larrossa, N., Avena, M., Rodriguez, A., Del Olmo, S., Bertucci, C., Muñoz, A., Castelló, E., Bazán, R. y Martínez, M. (2000). Estudios preliminares de la calidad de agua y sedimentos del embalse San Roque relacionados al proceso de eutroficación. Memorias XVIII Congreso Nacional Del Agua, Santiago Del Estero, Argentina.

Rodríguez, M. I., Busso, F., Bustamante, M. A., Ruibal Conti, A. L., Ruiz, M. y Angelaccio, C. M. (2005). Floraciones de algas en el embalse San Roque (Córdoba). Memorias XX Congreso Nacional del Agua y III Simposio de Recursos Hídricos del Cono Sur. Mendoza.

Rodríguez, M. I., Ruiz, M., Nadal, N., Halac, S., Olivera, P., Busso, F. y Bonfanti, E. (2013). Monitoreo y calidad de agua del embalse San Roque (Cba., Argentina). Memorias XXIV Congreso Nacional del Agua (CONAGUA). San Juan. Argentina.

Rodríguez-Zambrano, A. P. y Aranguren-Riaño, N. J. (2014). Comunidad planctónica de un embalse con alta tensión ambiental: La Playa, cuenca alta del río Chicamocha (Tuta, Boyacá), Colombia. Biota Colombiana, 15 (2): 95-110.

Ruibal Conti, A. L., Bustamante, A., Granero, M., López, F., Girbal, A., Lammel, E., Simonin, M. y Busso, F. (1999). Estudio de la evolución de la calidad de agua del embalse San Roque (Córdoba) asociado al desarrollo de floraciones de Ceratium. Memorias Congreso Argentino de Gran Presas y Aprovechamiento Hidroeléctricos. San Martín de Los Andes. Argentina.

Sabater, S., Sabater, F. y Armengol, J. (1988). Relationships between diatom assemblages and physico-chemical variables in the River Ter (N.E. Spain). Internationale Revue gesamten Hydrobiologie, 73: 171-179.

Schlegel, I., Koschel, R. y Krienitz, L. (1998). On the occurrence of Phacotus lenticularis (Chlorophyta) in lakes of different trophic state. Hydrobiologia, 69: 353- 361.

Shannon, C. E. y Weaver, W. (1949). The Mathematical Theory of Communication. Urbana. 
Chicago, III. Univ. Illinois Press, London.

Silva, L. C., Leone, I. C., Santos-Wisniewski, M. J., Peret, A. C. y Rocha, O. (2012). Invasion of the dinoflagellate Ceratium furcoides (Levander) Langhans 1925 at tropical reservoir and its relation to environmental variables. Biota Neotropica, 12(2): 93-100.

Sournia, A. E. (1978). Phytoplankton manual. Monographs on Oceanographic Methodology. 6. UNESCO. Paris.

Stumm, W. y Morgan, J.J. (1981). Aquatic Chemistry: An Introduction Emphasizing Chemical Equilibria in Natural Waters. WileyInterscience, New York.

Subsecretaria de Recursos Hídricos. (2008). Plan Nacional Federal de los Recursos Hídricos. Coordinado por Andrés Rodríguez $1^{\circ}$ ed. Buenos Aires. Ministerio de Planificación Federal.

Sun, J. y Liu, D. (2003). Geometric models for calculating cell biovolume and surface area for phytoplankton. Journal of Plankton Research, V 25: 1331-1346.

Temponeras, M., Kristiansen, J. y Moustaka-Gouni, M. (2000). Seasonal variation in phytoplankton composition and physicalchemical features of the shallow Lake Doirani, Macedonia, Greece. Hydrobiologia, 424: 109-122.
Winsborough, B. y Golubić, S. (1987). The role of diatoms in stromatolite growth: two examples from modern freshwater settings. Journal of Phycology. 23: 195- 201.

Zalocar de Domitrovic, Y. (1999). Etructura y dinámica del fitoplancton en la cuenca del eje potámico Paraguay-Paraná (Argentina). Tesis doctoral, Universidad Nacional de Córdoba.

\section{Tipo de Publicación: ARTICULO.}

Trabajo recibido el 05/06/2020 y aprobado para su publicación el 27/07/2020.

\section{COMO CITAR}

Daga, I. C., Fernández Belmonte, M. C. y Reyna, S. M. (2020). Composición algal y bioindicadores de calidad de agua. Caso de estudio: Embalse San Roque, Córdoba. Argentina. Cuadernos del CURIHAM. 26: 111. DOI: https://doi.org/10.35305/curiham.v26i0.143

Este es un artículo de acceso abierto bajo licencia: Creative Commons Atribución - No Comercial Compartir Igual 4.0 Internacional (CC BY-NC-SA 4.0) (https://creativecommons.org/licenses/by-ncsa/4.0/deed.es) 